Fev. Saúlc pübl. S. 1'uulo

$11: 551-60,197 \pi$

\title{
PESO AO NASCER DE FILHOS DE UM GRUPO DE MULHERES NORMAIS
}

\author{
Ana Cristina d'Andretta Tanaka * \\ Arnaldo Augusto Franco de Siqueira* \\ Augusta Thereza de Alvarenga * \\ Pedro Augusto Marcondes de Almeida* \\ Cvro Ciari Junior"
}

R.SPU-B/286

Tanaka, A. C. d.A. et al. Peso ao nascer de filhos de um grapo de mulheres normais. Rev. Saúde públ., S. Palllo, 11:551-60, 1977.

RESUMO: Estudou-se o comportamento do peso do recém-nascido procurando compará-lo com as curvas construidas em paises desenvolvidos e muito utilizadas em nosso meio. A partir de uma "Curva Ponderal de Gestantes Normais" foram selecionadas 996 mulheres com ausência de patologias próprias ou associadas à gravidez, subnutricăo e obesidade. O trabalho demonstrou que os pesos dos recém-nascidos aumentaram com o decorrer da idade gestacional, comecando, porém, a decair a partir da $41^{\circ}$ semana, explicáveis por problemas proprios do final da gestaçáo. Comparando-se estes resultados com os já existentes na literatura, pode-se verificar aue sua distribuiça foi semelhante ou superior aos mesmos, o que permite constatar que o peso co nascer das criancas de malheres normais selecionadas para o estado, foi semelhante ao de recém-nascidos de paises desenvolvidos.

Unitermos: Peso ao nascer. Idade gestacional. Recém-nascido.

INTRODUCAO

Una das grandes prencupaçós dus obstetras e pediatras, atualmente, é peso que irá ter o feto ao nascer. Estudos atuais mostram que o baixo peso leva 0 recém-nascido a dois tipos de risco. $\quad 0$ primeiro, que é o imediato, predispóe a uma maior mortalidade neonatal. $O$ segundo, mais tardio, irá refletir diretamente no crescimento e desenvolvimento da criança e em seu desempenho neuropsicomotor.

De acordo con a idacle gestacional, podese classificar os recém-nascidos em pré- termo, termu e pós-termo. ${ }^{1}$ Ainda, segund" Sweet, an se relacionar esta classificação com o peso ao nascer, pode-se por sua vez agrupá-los em:

- Apropriado para a idade gestacional (AIG)

- Pegueno para a idade gestacional ( $\mathrm{P} \mid \mathrm{G})$

-.- Grande para a idade gestacional (GIO)

Infelizmente, por ser a idade gestacional muitas vezes de difícil obtenção, em paises

* Do Departamento de Saúde Matemo-Infantil da Faculdade de Saúde Pública cia USP - Av. Dr. Arnaldo, 715 - Săo Paulo, SP - Brasil. 
TANAKA, A. C. d'A. et al. Peso ao nascer de $\mathrm{f}$ ilhos de um grupo de mulheres normais. Rev.

Saude publ., S. Paulo, 11:551-60. 1977.

em desenvolvimento, em virtude da qualidade dos dados disponiveis, assim como do nivel de informação da mulher, no Brasil tem se utilizado, para avaliar o peso do recém-nascido, curvas de peso ao nascer por idade gestacional, construidas em paises desenvolvidos. A mais utilizada em nosso meio é a curva de Lubchenco e col. ${ }^{5}$ (1963) ou a curva de Battaglia \& Lubchenco ${ }^{1}$ (1967).

Estudos que em nosso meio utilizaram a curva de Lubchenco mostram que os nossos recém-nascidos estão com seus pesos abaixu dos dessa curva: Para a construção da curva de Lubchenco e col. "foram utilizados pesos de crianças nascidas em Denver (Colurado). Essa cidade situa-se a 10.000 pés acina do nivel do mar; como se sabe que a altitude influi negativamente no peso do nascituro, os valores expressus nessa curva são ainda inferiores aos de outras semelhantes, como é o caso da de Thomson e col. (1968) e da Usher e Melean 10 (1969) e a de Gruenwald 3 (1966).

Assin visto, parece que nossos recénnascidos possuem pesus inferiores aos dus de paises desenvolvidos por problemas puramente genéticos e étnicos.

No entanto, estudos recentes " mostram yue a desnutrição materna ou o peso inadequado da mulher para sua estatura (subnutrição) podem ocasionar a desnutrição intra-uterina, com consequiente diminuição do peso ao nascer.

Com esta preocupação, este trabalho estuda os pesos de recém-nascidos de mulheres normais e os compara com diversas curvas de paises desenvolvidos para observar seu comportamento e com isso verificar se realmente os recém-nascidos, filhos de mulheres normais, possuem peso inferior aos de crianças de países desenvolvidos.

\section{MATERIAL E MÉTODOS}

Foram estudados três grupos de gestantes, sendo um grupo que recebia atenção pré-natal no Centro de Saúde Geraldo de Paula Souza da Faculdade de Saúde Públi- ca da USP, outro do Serviço de Pré-Natal da Escola Paulista de Medicina e o terceiro de um consultório particular que atende a gestantes de renda elevada, utilizando-se registros clínicos da ficha de pré-natal, que continham dados sobre a gestaçāo e o peso do recém-nascido.

Foram assim incluidas para estudo 1.500 gestantes que não apresentavam patologia própria ou associada à gestação.

A seguir, foram essas gestantes classificadas em subnutridas, normais ou obesas, una vez aplicadas as curvas de ganho de peso de gestantes, desenvolvidas no Departamento de Saúde Materno-Infantil da Faculdacle de Saude Publica da USP.'

Finalmente, as gestantes "subnutridas" e "obesas" foran excluidas, e restaram 996 gestantes sadias e normais quanto às curvas de ganho de peso.

Os pesos ao nascer de seus filhos foram registrados e distribuidos segundo a idade gestacunal, desde 35 até 43 semanas. Idades abaix, de 35 e acima de 43 semanas foram desprezadas, pelo número muito pequeno de casos.

\section{RESULTADOS E DISCUSSAO}

Será inicialmente discutido o peso do recém-nascido e depois será feita a comparação com as diversas curvas já citadas.

\section{Peso do recém-nascido}

Como era esperado, o peso dos recémnascidos aumentou com a idade gestacional. No entanto, ao atingir 42 semanas o peso começa a diminuir, fato este que diverge da maioria dos autores $(3,4,5,9)$. Isto provavelmente se deve a problemas próprios do fim da gestação (envelhecimento da placenta ou dificuldade de aporte anexial ou até mesmo por hábito alimentar da gestante, que começa a se alimentar de forma diferente após atingir a data provável do parto). Esses dados podem ser vistos na Tabela 1. 
TANAKA, A. C. d'A, et al. Peso ao nascer de filhos de um grupo de mulheres normais. Rev. Saude públ., S. Paulo, 11:551-60, 1977.

TABELA 1

Média de peso ao nascer por idade gestacional para cada sexo e para todos os recém-nascidos em estudo. São Paulo, 1977.

\begin{tabular}{c|c|c|c}
\hline Média de peso $(g)$ & Mem. & Masc. & $\begin{array}{c}\text { Ambos os } \\
\text { sexos }\end{array}$ \\
\hline Idade gestacional & & & 2.678 \\
\hline 35 & 2.672 & 2.945 & 2.856 \\
36 & 2.757 & 3.034 & 2.941 \\
37 & 2.850 & 3.089 & 3.078 \\
38 & 3.069 & 3.242 & 3.169 \\
39 & 3.103 & 3.411 & 3.303 \\
40 & 3.198 & 3.440 & 3.371 \\
41 & 3.310 & 3.464 & 3.283 \\
42 & 3.173 & & \\
\hline
\end{tabular}

A Tabela 2 e as Figs. 1 e 2 mostram que os percentis de peso do recém-nascido para todas as crianças e para cada sexo individualmente se comportam de maneira diferente. Os recém-nascidos de sexo feminino, como era de se esperar, possuem valores inferiores aos do sexo masculino.

\section{Curva de peso do recém-nascido}

Ao se comparar a curva dos recém-nascidos em estudo com as construídas em países desenvolvidos pode-se observar na Tabela 3 e Fig. 3:

2.1. A média de peso por idade gestacional dos nossos recém-nascidos está acima dos valores da curva de Lubchenco e col. ${ }^{5}$; exceção se faz à idade gestacional de 42 semanas, fato este que foi discutido acima.

2.2. Ao compará-la com a curva de Gruenwald 3 observa-se que elas se entrelaçam, isto é, até 38 semanas ela está acima dos valores de
Gruenwald, na $39^{a}$ semana ela está abaixo, e na $40^{2}$ semana de gestação acima da de Gruenwald; ocorre de novo um declínio na idade gestacional de 42 semanas.

2.3. Em relação à de Thomson ${ }^{9}$, a curva em estudo situa-se abaixo de seus valores.

2.4. Quanto à de Usher e Melean ${ }^{10}$, no entanto, a curva elaborada tem seus valores acima da mesma até a idade gestacional de 37 semanas, ocorrendo, após este periodo, uma mudança de sua posição. É interessante notar que embora se verifique diferenças de valores, a tendência de ambas é a mesma uma vez que possuem um sentido ascendente até a $41^{*}$ semana, verificandose aí uma queda característica.

Pela análise verifica-se que os recém-nascidos neste estudo possuem uma distribuição de peso, por idade gestacional, semelhante ou superior às curvas encontradas 


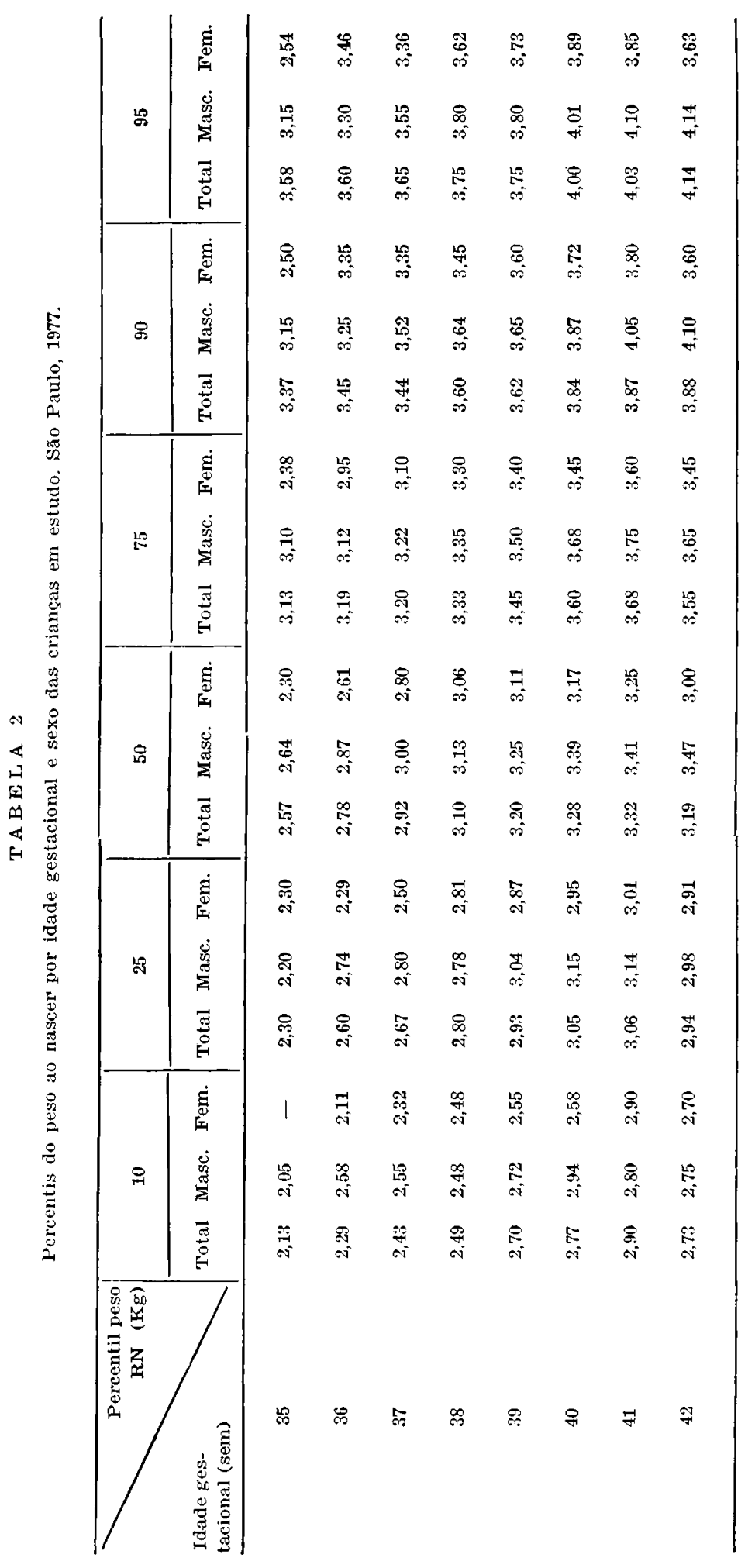


TANAKA, A. C. d'A. et ai. Peso ao nascer de filhos de um grupo de mulheres normais. Rev. Saúde públ., S. Paulo, 11:551-60, 1977.

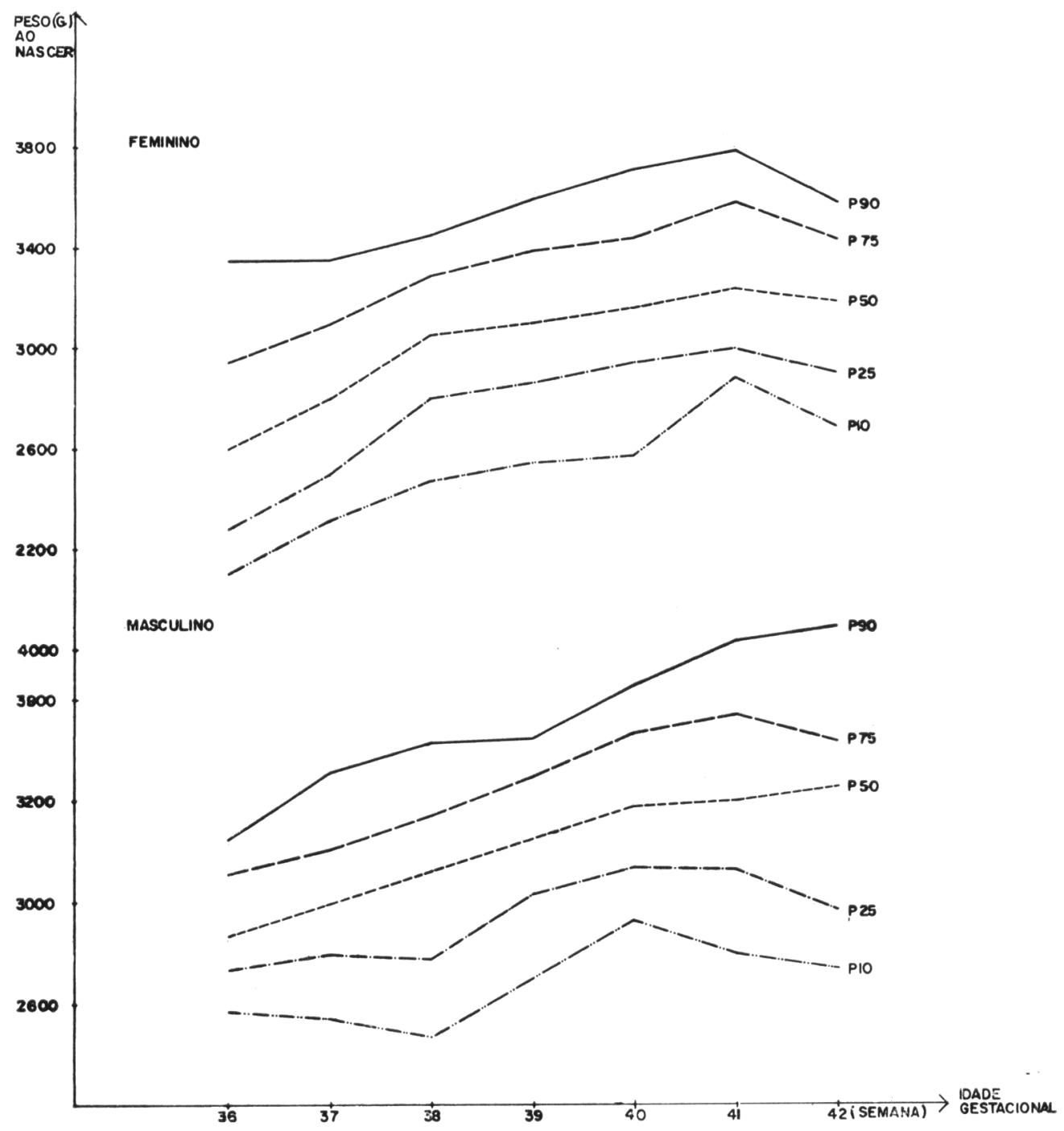

Fig. 1 - Percentil do peso ao nascer por idade gestacional e por sexo do grupo das crianças em estudo. São Paulo, 1977. 
TANAKA, A. C. d'A. et al. Peso ao nascer de $f$ lhos de um grupo de mulheres normais. Rev. Saúde públ., S. Paulo, 11:551-60, 1977.

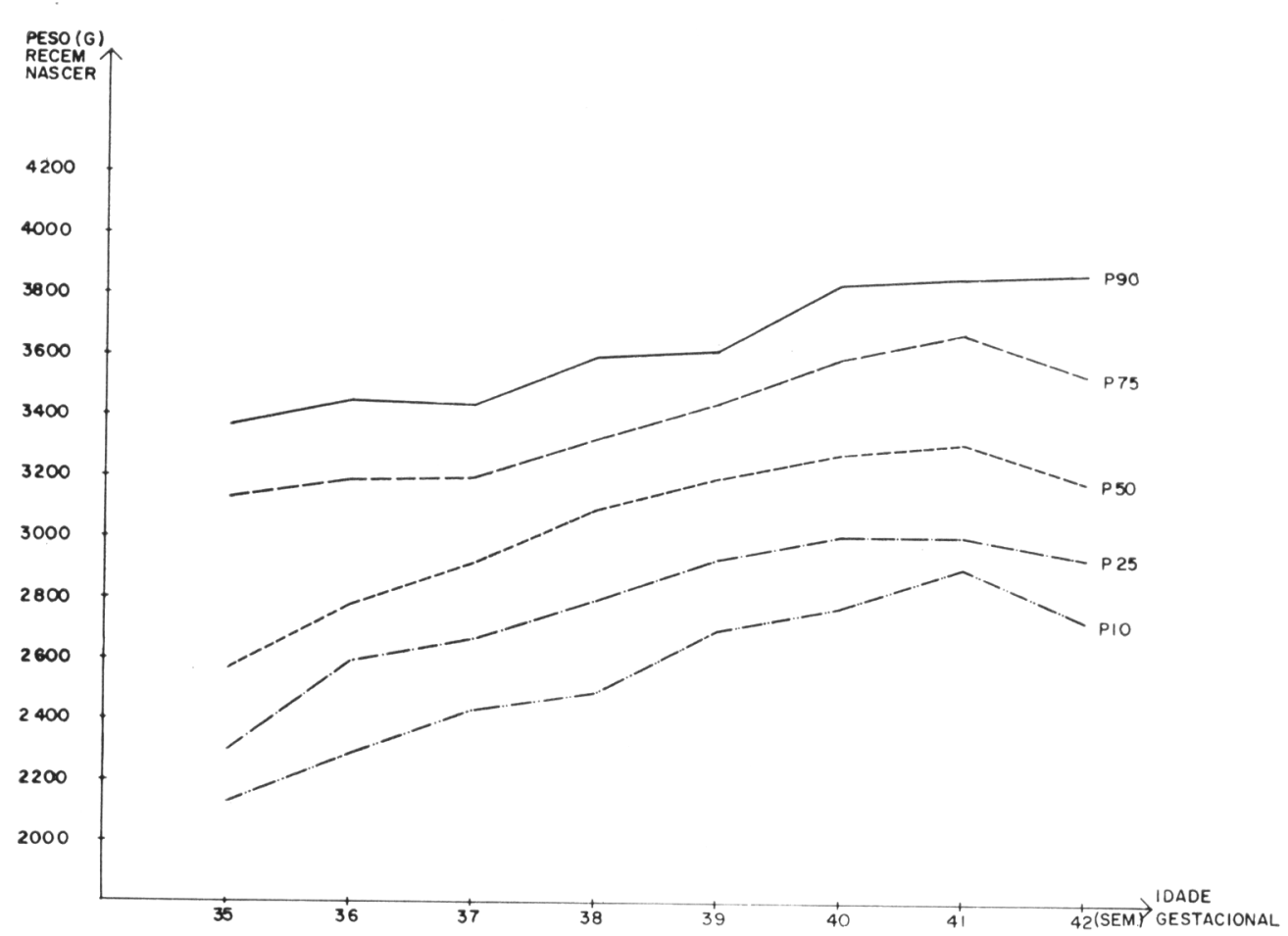

Fig. 2 - Percentil do peso ao nascer por idade gestacional do grupo das crianças em estudo. São Paulo, 1977.

T A B E L A 3

Média de peso ao nascer por idade gestacional de Lubchenco e col., Gruenwald, Thomson e col., Usher e Melean e das crianças em estudo. São Paulo, 1977*

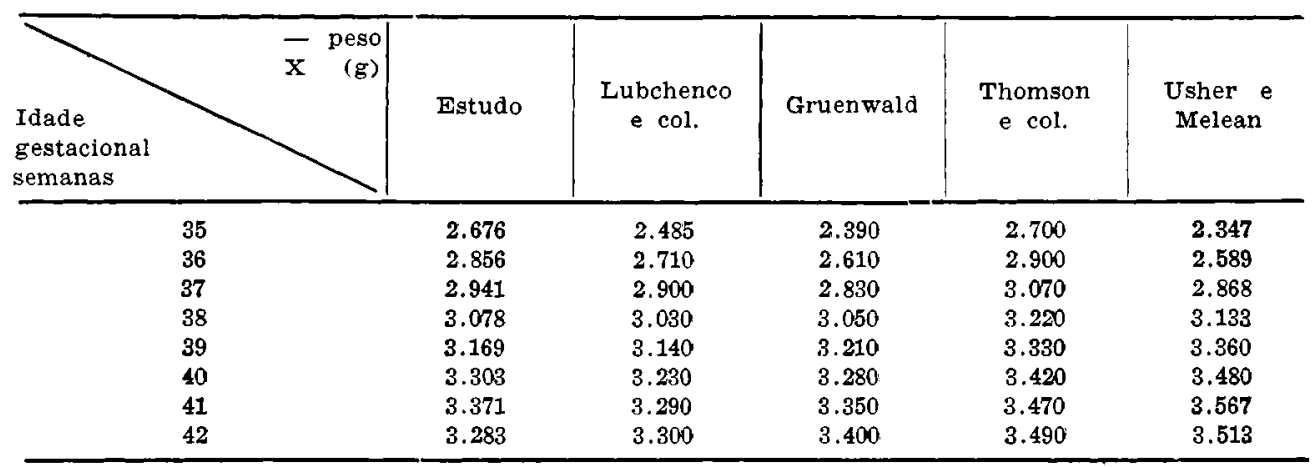

* Lubchenco e col.5 (1963)

Thomson e col.9 (1968)

Gruenwald 3 (1966)

Usher e Meleanıo (1969) 


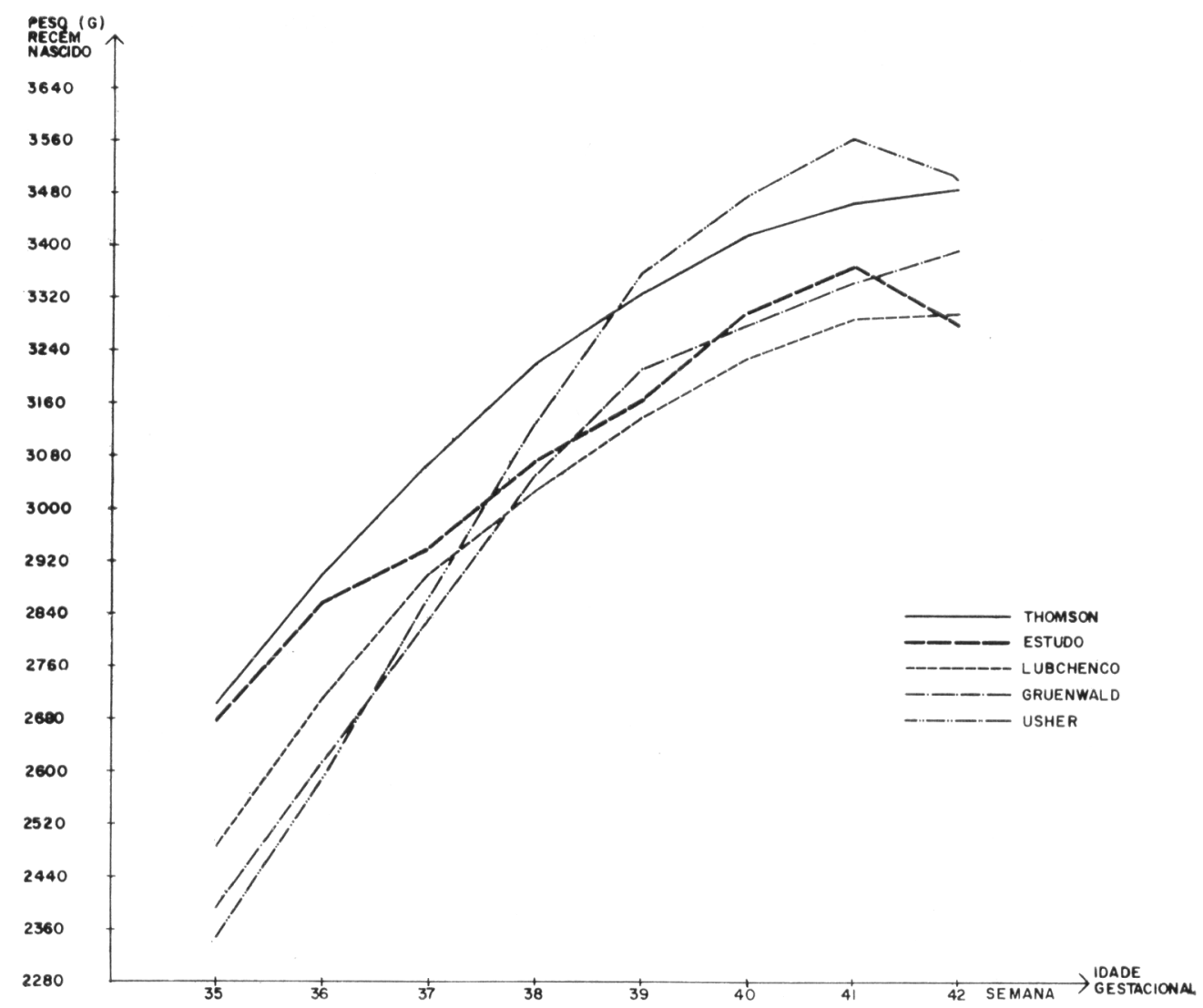

Fig. 3 - Curva de méđia de peso ao nascer por idade gestacional de Usher. Thomson. Gruenwald, Lubchenco e dos recém-nascidos em estudo. São Paulo, 1977.

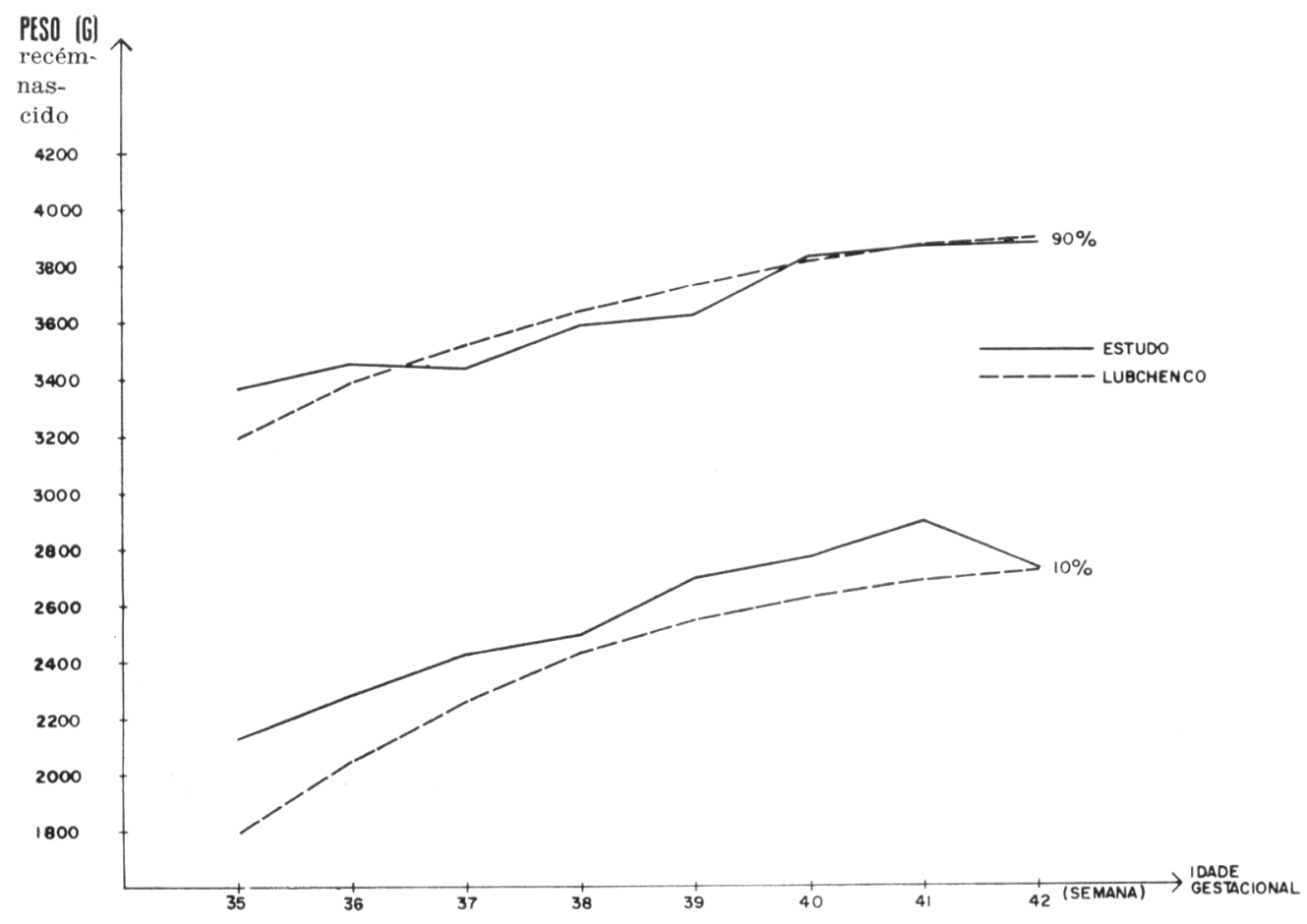

Fig. 4 - Percentil 10 e 90 de Lubchenco e das crianças em estudo. São Paulo, 1977. 


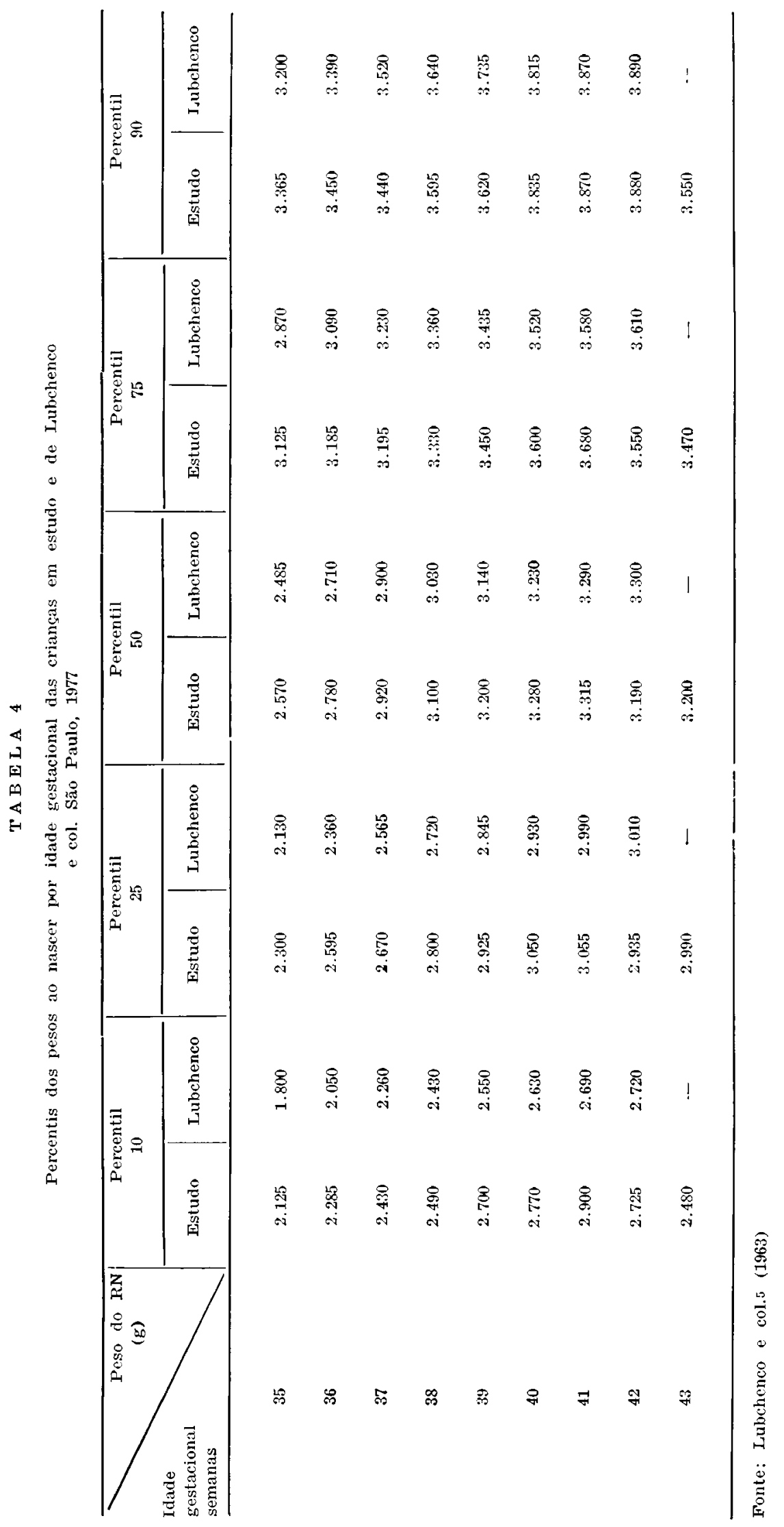


TANAKA, A. C. d'A. et al. Peso ao nascer de filhos de um grupo de mulheres normais. Rev. Saúde públ., S. Paulo, 11:551-60, 1977.

na literatura especializada sendo, portanto, o seu comportamento comparável aos dos recém-nascidos de países desenvolvidos.

A Tabela 4 e a Fig. 4 comparam os percentis 10 e 90 de Lubchenco e col. ${ }^{3}$ e dos recém-nascidos em estudo. Como é sabido, estes percentis 10 e 90 de Lubchenco e col. foram a base para a construção da curva de Battaglia e Lubchenco I para diagnóstico do risco fetal. Esta comparação permitiu-nos demonstrar que os nossos recém-natos estão sempre acima do percentil 10 de Lubchenco e col. e quase que superpostas ao 90 destes mesmos autores. Isto mostra uma vez mais que estes recémnascidos possuem pesos semelhantes ao de crianças de países desenvolvidos desde que fatores como gemelaridade, patologias próprias (toxemia e outras) ou associadas (diabetes, cardiopatia) à gestação e à subnutrição ou desnutrição materna, que alteram o peso do recém-nascido, não sejam considerados.

Apesar da nossa curva ser semelhante às curvas disponiveis e já aqui analisadas, não se superpõe a nenhuma delas, o que leva à necessidade de estudos que permitam a construção de uma tabela adaptada ao nosso meio para o diagnóstico do risco do recém-nascido.

\section{CON CLUSOES}

1. Ao se retirar o fator desnutrição os recém-nascidos filhos de mulheres normais possuem características de crescimento intra-uterino semelhantes aos de qualquer pais desenvolvido.

2. Este trabalho mostra que não se pode estudar isoladamente o fator peso do recém-nascido sen se levar em conta os fatores maternos, pois as condiçōes pré-natais (patologia, estado nutricional e nível sócio-econônico) influenciam diretamente o peso do recém-nascido. Não se levando estes dados em consideração, distorsões neste tipo de estudo poderão ocorrer.

TANAKA, A. C. d'A. et al. [Birth weight of infants in a group of normal women.]

Rev. Saúde públ., S. Paulo, 11:551-60, 1977.

ABSTRACT: In this paper the authors studied the birth weight of the children of 996 pregnant women in good health. These women had been classified as in adequated mutritional status by the application of a "Weight Curve of Pregnant Women". Birth weight increased with gestational age until $41^{\text {st }}$ week of gestation, decreasing afterwards. Comparing our birth weight curve with those of Gruenwald, Labchenco, Usher $e$ Thomson, we can say that the distribution of birth weight of our children was similar or higher than those curves. These facts allow us to conclude that birth weights of products of normal pregnant women of this stady were the same of newborns of developed countries.

UNITERMS: Birth weight. Gestational age. Infant newborn. 
TANAKA, A. C. d'A. et al. Peso ao nascer de filhos de um grupo de mulheres normais. Rev. Saúde públ., S. Paulo, 11:551-60, 1977.

\section{REFERENCIAS BIBLIOGRAFICAS}

1. BATTAGLiA, F. \& LUBCHENCO, L. A practical classification of newborn infants by weight and gestational age. J. Pediat., $71: 159-63,1971$.

2. COLANERI, $C$, et al apud VIEGAS, D. \& DOMINGUES, L. C. Conceito atual do recém-nascido. Clin. pediat., 1(1):17-25, 1976.

3. GRUENWALD, P. Growth of the human fetus. I. Normal growth and its variation. Amer. J. Obstet. Gynec, 94. $1112-9,1966$.

4. LINDELL, A. Prolonged pregnancy, Acta obstst. gynec. scand., 35:136-63, 1956.

5. LUBCHENCO, $L$. $O$. et al. Intrauterine growth as estimated from liveborn birth-weight data at 24 to 42 weeks of gestation. Pediatrics, 32 :79,3-800. 1963.

6. SIQUEIRA, A. A. F, de et al. Influencia da altura e ganho de peso maternos e da idade gestacional sobre o peso do recém-nascido: estudo de 3 grupos de gestantes normais. Rev. Saúde públ., S. Paulo, 9:331-42, 1975

7. SIQUEIRA, A. A. F, de et al. A utilização de uma curva ponderal de gestantes normais no diagnóstico de desnutrição intra-uterina. Rev. Saúde pübl., $\mathrm{S}$. Paulo, 9:441-568, 1975.

8. SWEET, A. Y. Clasificacion del reciennascido de bajo peso. In: KLAUSS. M. H. \& FANAROFF A. A. Asistencia del recieranascido de alto riesgo. Buenos Aires, Ed. Medica Panamericana, 1977. p. 69-102.

9. THOMSON, A. M. et al. The assessment of fetal growth. J. Obstet. Gynaec. Brit. Cwith, 75:903-16, 1968.

10. USHER, R. \& MELEAN, F. - Intrauterine growth of live-born Causasian infants at sea level: 7 dimensions of infants born between 25 and 44 weeks of gestation J. Pediat., 74:901-10, 1969.

Recebido para publicação em 30/05/19\%\% A provado para publicação em 14/0r/197\% 